\title{
Additive-error fine-grained quantum supremacy
}

\author{
Tomoyuki Morimae ${ }^{1,2}$ and Suguru Tamaki ${ }^{3}$ \\ ${ }^{1}$ Yukawa Institute for Theoretical Physics, Kyoto University, Kitashirakawa Oiwakecho, Sakyoku, Kyoto 606-8502, Japan \\ 2 JST, PRESTO, 4-1-8 Honcho, Kawaguchi, Saitama, 332-0012, Japan \\ ${ }^{3}$ School of Social Information Science, University of Hyogo, 8-2-1, Gakuennishi-machi, Nishi-ku, Kobe, Hyogo 651-2197, \\ Japan
}

It is known that several sub-universal quantum computing models, such as the IQP model, the Boson sampling model, the one-clean qubit model, and the random circuit model, cannot be classically simulated in polynomial time under certain conjectures in classical complexity theory. Recently, these results have been improved to "fine-grained" versions where even exponential-time classical simulations are excluded assuming certain classical fine-grained complexity conjectures. All these fine-grained results are, however, about the hardness of strong simulations or multiplicative-error sampling. It was open whether any fine-grained quantum supremacy result can be shown for a more realistic setup, namely, additive-error sampling. In this paper, we show the additive-error fine-grained quantum supremacy (under certain complexity assumptions). As examples, we consider the IQP model, a mixture of the IQP model and $\log$-depth Boolean circuits, and Clifford $+T$ circuits. Similar results should hold for other sub-universal models.

\section{Introduction}

Classically sampling output probability distributions of sub-universal quantum computing models is known to be impossible under certain classical complexity conjectures. The depth-four model [1], the Boson Sampling model [2], the IQP model [3, 4], the one-clean qubit model [5, 6, 7, 8, 9], the HC1Q model [10], and the random circuit model [11, 12, 13] are known examples. These results prohibit only polynomial-time classical sampling, but recently, impossibilities of some exponential-time classical simulations have been shown based on classical fine-grained complexity conjectures $[14,15,16,17,18,19]$.

These "fine-grained" quantum supremacy results are, however, only for exact computations (i.e., strong simulations) or multiplicative-error sampling of output probability distributions. Here, we say that a quantum probability distribution $\left\{p_{z}\right\}_{z}$ is classically sampled in time $T$ within a multiplicative error $\epsilon$ if there exists a classical $T$-time probabilistic algorithm that outputs $z$ with probability $q_{z}$ such that $\left|p_{z}-q_{z}\right| \leq \epsilon p_{z}$ for all $z$. It was open whether fine-grained quantum supremacy can be shown for additive-error sampling. Here, we say that a quantum probability distribution $\left\{p_{z}\right\}_{z}$ is classically sampled in time $T$ within an additive error $\epsilon$ if there exists a classical $T$-time probabilistic algorithm that outputs $z$ with probability $q_{z}$ such that $\sum_{z}\left|p_{z}-q_{z}\right| \leq \epsilon$. Additive-error sampling is more realistic for medium-size noisy quantum computers, and therefore theoretically showing quantum supremacy for additive-error sampling is important for the nearterm experimental demonstrations of quantum supremacy.

In this paper, we show additive-error fine-grained quantum supremacy based on certain classical fine-grained complexity conjectures. As examples, we consider the IQP model (Sec. 2), a mixture of the IQP model and log-depth Boolean circuits (Sec. 3), and Clifford $+T$ circuits (Sec. 4). Similar results should hold for other sub-universal models.

Tomoyuki Morimae: tomoyuki.morimae@yukawa.kyoto-u.ac.jp

Suguru Tamaki: tamak@sis.u-hyogo.ac.jp 
The second result (IQP plus log-depth Boolean circuit) needs more complicated quantum circuit than the first one, but the conjecture seems to be more reliable. The first and second results are about the scaling for the number of qubits, while the third result is about the scaling for the number of $T$ gates.

The standard proof technique of additive-error quantum supremacy [2, 4], namely, the combination of Markov's inequality, Stockmeyer's theorem, and the anti-concentration lemma, cannot be directly used for fine-grained quantum supremacy, because Stockmeyer's theorem is a result for polynomial-time probabilistic computing. (Markov's inequality and the anti-concentration lemma can be used because they are independent of the time complexity.) In order to show fine-grained additive-error quantum supremacy, we derive a "fine-grained version" of Stockmeyer's theorem. Our results, for the first time, demonstrate that the standard proof technique of additive-error quantum supremacy can be extended to exponential-time hardness.

Because there is a gap between the upper-bound and the lower-bound, the results have a potential to be improved or sharpened. The upper-bound can be improved by faster simulations, and the lower-bound can be improved by improving the reduction or by introducing other conjectures. Furthermore, possible ways are extensions of the MA algorithm to refute SETH [20], and the fine-grained reductions from approximate counting to decision [21].

Note: After uploading this paper on arXiv, authors of Ref. [14] told us that they also show independently additive-error fine-grained quantum supremacy results. (Their additive-error results are added in their latest version.) They consider an exponential-time version of the SBP $\neq \mathrm{SBQP}$ conjecture. (If SBQP $=\mathrm{SBP}$, the polynomial-time hierarchy collapses to the second level.) At this moment, we do not know how their conjecture and ours are related.

\section{$2 \mathrm{IQP}$}

In this section, we show additive-error fine-grained quantum supremacy of the IQP model. The IQP model is defined as follows.

Definition 1 An $N$-qubit IQP model is the following quantum computing model:

1. The initial state is $\left|0^{N}\right\rangle$. (Here, $\left|0^{N}\right\rangle=|0\rangle^{\otimes N}$.)

2. $H^{\otimes N}$ is applied, where $H$ is the Hadamard gate.

3. $Z$-diagonal gates (such as $e^{i \theta Z}, Z, C Z$, and $C C Z$ ) are applied. (In this paper, we consider only $Z, C Z$, and $C C Z$.)

4. $H^{\otimes N}$ is applied.

5. All qubits are measured in the computational basis.

Let us consider an $n$-variable degree-3 polynomial, $f:\{0,1\}^{n} \rightarrow\{0,1\}$, over $\mathbb{F}_{2}$ defined by

$$
f\left(x_{1}, \ldots, x_{n}\right) \equiv \sum_{i=1}^{n} \alpha_{i} x_{i}+\sum_{i>j} \beta_{i, j} x_{i} x_{j}+\sum_{i>j>k} \gamma_{i, j, k} x_{i} x_{j} x_{k}
$$

for any $x \equiv\left(x_{1}, x_{2}, \ldots, x_{n}\right) \in\{0,1\}^{n}$, where $\alpha_{i}, \beta_{i, j}, \gamma_{i, j, k} \in\{0,1\}$. If we say that we randomly choose $f$, it means that we randomly choose each $\alpha_{i}, \beta_{i, j}, \gamma_{i, j, k}$ uniformly and independently.

The conjecture on which additive-error fine-grained quantum supremacy of the IQP model is based is stated as follows.

Conjecture 1 Let $f$ be an n-variable degree-3 polynomial over $\mathbb{F}_{2}$. Let us define

$$
\operatorname{gap}(f) \equiv \sum_{x \in\{0,1\}^{n}}(-1)^{f(x)}
$$

There exist positive constants $a$ and $n_{0}$ such that for every $n>n_{0}$ the following holds. Computing $[\mathrm{gap}(f)]^{2}$ within a multiplicative error $u$ for at least $v$ fraction of $f$ cannot be done with a classical probabilistic $O^{*}\left(2^{\text {an }}\right)$-time algorithm that makes queries of length $O\left(2^{\text {an }}\right)$ to an NTIME $\left.n^{2}\right]$ oracle with a success probability at least $w$. Here, $u, v, w$ are certain constants. $\left(O^{*}\right.$ means that the polynomial factor is ignored.) 
Here, the oracle query is the standard one: there is a separate oracle tape and answers can be returned from the oracle instantaneously. Note that the parameters $u, v, w$ can be adjustable to some extent. (See the proof.) We do not know whether this conjecture is true or false, but at least at this moment we do not know how to refute it. (For more discussions, see Sec. 5.1).

Based on Conjecture 1, we show the following result.

Theorem 1 If Conjecture 1 is true, then there exists an N-qubit IQP circuit whose output probability distribution cannot be classically sampled in $O\left(2^{a N}\right)$-time within a certain constant additive error $\epsilon$.

For simplicity, we consider degree-3 polynomials, but it is clear from the following proof that a similar result holds for degree- $k$ polynomials for any constant $k \geq 3$. (The anti-concentration lemma holds for any degree- $k$ polynomial with $k \geq 2$, but the degree- 2 case is classically simulatable because it is a Clifford circuit, so $k \geq 3$ is necessary.) If we consider Conjecture 1 for degree- $k$ polynomials, it becomes more stable for larger $k$ [22].

Proof of Theorem 1. Given an $n$-variable degree-3 polynomial $f$, we can construct an $n$-qubit IQP circuit such that the probability $p_{z}(f)$ of outputting $z \in\{0,1\}^{n}$ satisfies

$$
p_{z}(f)=\frac{\left(g a p\left(f_{z}\right)\right)^{2}}{2^{2 n}},
$$

where

$$
f_{z}\left(x_{1}, \ldots, x_{n}\right) \equiv f\left(x_{1}, \ldots, x_{n}\right)+\sum_{i=1}^{n} z_{i} x_{i} .
$$

Assume that there exists a $T$-time classical probabilistic algorithm that outputs $z \in\{0,1\}^{n}$ with probability $q_{z}(f)$ such that

$$
\sum_{z \in\{0,1\}^{n}}\left|p_{z}(f)-q_{z}(f)\right| \leq \epsilon
$$

for a certain $\epsilon$ and any $f$. From Markov's inequality,

$$
\operatorname{Pr}_{z}\left[\left|p_{z}(f)-q_{z}(f)\right| \geq \frac{\epsilon}{2^{n} \delta}\right] \leq \delta
$$

for any $f$ and $\delta>0$. According to the fine-grained Stockmeyer's theorem (see Appendix), a classical $O^{*}(T)$-time probabilistic algorithm that makes queries of $O(T)$ length to the NTIME $\left[n^{2}\right]$ oracle can compute $\tilde{q}_{z}(f)$ such that

$$
\left|q_{z}(f)-\tilde{q}_{z}(f)\right| \leq \xi q_{z}(f)
$$

where

$$
\xi \equiv \frac{2^{\frac{1}{\alpha}}-2^{-\frac{1}{\alpha}}}{2},
$$

for any $f$, integer $\alpha \geq 1$, and $z \in\{0,1\}^{n}$, with a success probability at least $w$. Due to the anti-concentration lemma [4],

$$
\operatorname{Pr}_{z, f}\left[p_{z}(f) \geq \frac{\tau}{2^{n}}\right] \geq \frac{(1-\tau)^{2}}{3}
$$

for any $0<\tau<1$. 
Therefore we have

$$
\begin{aligned}
\left|p_{z}(f)-\tilde{q}_{z}(f)\right| & \leq\left|p_{z}(f)-q_{z}(f)\right|+\left|q_{z}(f)-\tilde{q}_{z}(f)\right| \\
& \leq\left|p_{z}(f)-q_{z}(f)\right|+\xi q_{z}(f) \quad(\text { with a success probability at least } w \text { for each } f \text { and } z) \\
& \leq\left|p_{z}(f)-q_{z}(f)\right|+\xi\left(p_{z}(f)+\left|p_{z}(f)-q_{z}(f)\right|\right) \\
& =\xi p_{z}(f)+\left|p_{z}(f)-q_{z}(f)\right|(1+\xi) \\
& \leq \xi p_{z}(f)+\frac{\epsilon}{2^{n} \delta}(1+\xi) \quad(\text { for at least } 1-\delta \text { fraction of } z) \\
& \leq \xi p_{z}(f)+\sigma p_{z}(f)(1+\xi) \quad\left(\text { for at least } \frac{\left(1-\frac{\epsilon}{\sigma \delta}\right)^{2}}{3} \text { fraction of }(z, f)\right) \\
& =p_{z}(f)(\sigma+(1+\sigma) \xi) \\
& =p_{z}(f) u(\text { We take } u \equiv \sigma+(1+\sigma) \xi) .
\end{aligned}
$$

If we take $\epsilon$ and $\delta$ such that $-\delta+\frac{1}{3}\left(1-\frac{\epsilon}{\sigma \delta}\right)^{2}=v$, the above inequality is correct for at least $v$ fraction of $(z, f)$. Hence, we obtain

$$
\left|\left(\operatorname{gap}\left(f_{z}\right)\right)^{2}-2^{2 n} \tilde{q}_{z}(f)\right| \leq u\left(\operatorname{gap}\left(f_{z}\right)\right)^{2}
$$

for at least $v$ fraction of $(z, f)$. It means $(\operatorname{gap}(f))^{2}$ is computable within the multiplicative error $u$ for at least $v$ fraction of $f$, which contradict Conjecture 1 .

Note that $w$ can be arbitrary close to 1 , but $u$ is lower-bounded as $u \geq \frac{\epsilon}{1+\sqrt{3}}$, and $v$ is upperbounded as $v \leq 1-\frac{\epsilon}{u(1+\sqrt{3})}$.

\section{IQP plus log-depth Boolean circuit}

In this section, we show additive-error fine-grained quantum supremacy for the IQP plus log-depth Boolean circuit model.

Let us consider the following conjecture.

Conjecture 2 Let $f:\{0,1\}^{n} \rightarrow\{0,1\}$ be an n-variable degree-2 polynomial over $\mathbb{F}_{2}$, and $g$ : $\{0,1\}^{n} \rightarrow\{0,1\}$ be an $n$-variable log-depth Boolean circuit. Define

$$
g a p(f+g) \equiv \sum_{x \in\{0,1\}^{n}}(-1)^{f(x)+g(x)}
$$

There exist $g$, and positive constants a and $n_{0}$ such that for every $n>n_{0}$ the following holds. Computing $[\operatorname{gap}(f+g)]^{2}$ within a multiplicative error $u$ for at least $v$ fraction of $f$ cannot be done with a classical probabilistic $O^{*}\left(2^{\text {an }}\right)$-time algorithm that makes queries of length $O\left(2^{\text {an }}\right)$ to an NTIME $\left[n^{2}\right]$ oracle with a success probability at least $w$.

Conjecture 2 is "more stable" than Conjecture 1, because log-depth Boolean circuit is more general than constant-degree polynomials. For constant-degree polynomials, there is a non-trivial exponential time algorithm to count the number of solutions [23], but we do not know how to apply it to log-depth Boolean circuits. Furthermore, note that in Conjecture 2, the average case is considered only for $f$, and $g$ can be taken as the worst case one.

Based on Conjecture 2, we show the following result.

Theorem 2 If Conjecture 2 is true, then there exists an $N$-qubit poly $(N)$-size quantum circuit (consisting of an IQP circuit and a log-depth Boolean circuit) whose output probability distribution cannot be classically sampled in $O\left(2^{a N}\right)$-time within a certain constant additive error $\epsilon$.

Proof of Theorem 2. Given a log-depth Boolean circuit $g:\{0,1\}^{n} \rightarrow\{0,1\}$, we can construct an $(n+1)$-qubit poly $(n)$-size quantum circuit $U$ such that

$$
U(|x\rangle \otimes|0\rangle)=e^{i h(x)}|x\rangle \otimes|g(x)\rangle
$$

for any $x \in\{0,1\}^{n}$, where $h$ is a certain function whose detail is irrelevant here [24]. Let us consider the following circuit. 
1. The initial state is $\left|0^{n}\right\rangle \otimes|0\rangle$.

2. Apply $H^{\otimes n} \otimes I$ to obtain

$$
\frac{1}{\sqrt{2^{n}}} \sum_{x \in\{0,1\}^{n}}|x\rangle \otimes|0\rangle .
$$

3. Apply $U$ to obtain

$$
\frac{1}{\sqrt{2^{n}}} \sum_{x \in\{0,1\}^{n}} e^{i h(x)}|x\rangle \otimes|g(x)\rangle .
$$

4. Apply $Z$ on the last qubit to obtain

$$
\frac{1}{\sqrt{2^{n}}} \sum_{x \in\{0,1\}^{n}} e^{i h(x)}(-1)^{g(x)}|x\rangle \otimes|g(x)\rangle .
$$

5. Apply $U^{\dagger}$ to obtain

$$
\frac{1}{\sqrt{2^{n}}} \sum_{x \in\{0,1\}^{n}}(-1)^{g(x)}|x\rangle \otimes|0\rangle .
$$

6. Apply $Z$ and $C Z$ that correspond to $f$ to obtain

$$
\frac{1}{\sqrt{2^{n}}} \sum_{x \in\{0,1\}^{n}}(-1)^{g(x)+f(x)}|x\rangle \otimes|0\rangle .
$$

7. Apply $H^{\otimes n} \otimes I$ and measure the first $n$ qubits in the computational basis.

The probability of obtaining $z \in\{0,1\}^{n}$ is

$$
\begin{aligned}
p_{z}(f+g) & =\left|\frac{1}{2^{n}} \sum_{x \in\{0,1\}^{n}}(-1)^{g(x)+f(x)+\sum_{j=1}^{n} x_{j} z_{j}}\right|^{2} \\
& =\frac{\left(\operatorname{gap}\left(g+f_{z}\right)\right)^{2}}{2^{2 n}} .
\end{aligned}
$$

Assume that there exists a $T$-time classical probabilistic algorithm that outputs $z \in\{0,1\}^{n}$ with probability $q_{z}(f+g)$ such that

$$
\sum_{z \in\{0,1\}^{n}}\left|p_{z}(f+g)-q_{z}(f+g)\right| \leq \epsilon .
$$

From Markov's inequality,

$$
\operatorname{Pr}_{z}\left[\left|p_{z}(f+g)-q_{z}(f+g)\right| \geq \frac{\epsilon}{2^{n} \delta}\right] \leq \delta
$$

for any $f, g$, and $\delta>0$. According to the fine-grained Stockmeyer's theorem, a classical $O^{*}(T)$-time probabilistic algorithm that makes queries of length $O(T)$ to the NTIME $\left[n^{2}\right]$ oracle can compute $\tilde{q}_{z}(f+g)$ such that

$$
\left|q_{z}(f+g)-\tilde{q}_{z}(f+g)\right| \leq \xi q_{z}(f+g)
$$

where

$$
\xi \equiv \frac{2^{\frac{1}{\alpha}}-2^{-\frac{1}{\alpha}}}{2}
$$


for any $f, g$, integer $\alpha \geq 1$, and $z \in\{0,1\}^{n}$, with a success probability at least $w$. Due to the anti-concentration lemma [4]

$$
\operatorname{Pr}_{z, f}\left[p_{z}(f+g) \geq \frac{\tau}{2^{n}}\right] \geq \frac{(1-\tau)^{2}}{3}
$$

for any $0<\tau<1$.

Then we have

$$
\begin{aligned}
\left|p_{z}(f+g)-\tilde{q}_{z}(f+g)\right| & \leq\left|p_{z}(f+g)-q_{z}(f+g)\right|+\left|q_{z}(f+g)-\tilde{q}_{z}(f+g)\right| \\
& \leq\left|p_{z}(f+g)-q_{z}(f+g)\right|+\xi q_{z}(f+g) \\
& \leq\left|p_{z}(f+g)-q_{z}(f+g)\right|+\xi\left(p_{z}(f+g)+\left|p_{z}(f+g)-q_{z}(f+g)\right|\right) \\
& =\xi p_{z}(f+g)+\left|p_{z}(f+g)-q_{z}(f+g)\right|(1+\xi) \\
& \leq \xi p_{z}(f+g)+\frac{\epsilon}{2^{n} \delta}(1+\xi) \quad(\text { for at least } 1-\delta \text { fraction of } z) \\
& \leq \xi p_{z}(f+g)+\sigma p_{z}(f+g)(1+\xi) \quad\left(\text { for at least } \frac{\left(1-\frac{\epsilon}{\sigma \delta}\right)^{2}}{3} \text { fraction of }(z, f)\right) \\
& =p_{z}(f+g)(\sigma+(1+\sigma) \xi) \\
& =p_{z}(f+g) u(\text { We take } u \equiv \sigma+(1+\sigma) \xi) .
\end{aligned}
$$

If we take $\epsilon$ and $\delta$ such that $-\delta+\frac{1}{3}\left(1-\frac{\epsilon}{\sigma \delta}\right)^{2}=v$, the above inequality is correct for at least $v$ fraction of $(z, f)$, which contradict Conjecture 2 .

\section{Clifford plus $T$}

In this section, we finally show additive-error fine-grained quantum supremacy for Clifford $+T$ circuits. Let us consider the following conjecture.

Conjecture 3 Let $g:\{0,1\}^{n} \rightarrow\{0,1\}$ be a 3-CNF with $m$ clauses, and $f:\{0,1\}^{n} \rightarrow\{0,1\}$ be an $n$-variable degree-2 polynomial over $\mathbb{F}_{2}$. Define

$$
g a p(f+g) \equiv \sum_{x \in\{0,1\}^{n}}(-1)^{f(x)+g(x)} .
$$

There exist $g$, and positive constants a and $n_{0}$ such that for every $n>n_{0}$ the following holds. Computing $[\operatorname{gap}(f+g)]^{2}$ within a multiplicative error $u$ for at least $v$ fraction of $f$ cannot be done with a classical probabilistic $O^{*}\left(2^{\text {am }}\right)$-time algorithm that makes queries of length $O\left(2^{\text {am }}\right)$ to an NTIME $\left[n^{2}\right]$ oracle with a success probability at least $w$.

Based on Conjecture 3, we show the following result.

Theorem 3 If Conjecture 3 is true, then there exists a quantum circuit over Clifford gates and $t T$ gates whose output probability distribution cannot be classically sampled in $O\left(2^{\frac{a(t+14)}{42}}\right)$-time within a certain constant additive error $\epsilon$.

Proof of Theorem 3. Given a 3-CNF $g:\{0,1\}^{n} \rightarrow\{0,1\}$, we can construct a quantum circuit $U$ such that

$$
U\left(|x\rangle \otimes\left|0^{\xi}\right\rangle\right)=|g(x)\rangle \otimes|j u n k(x)\rangle
$$

for any $x \in\{0,1\}^{n}$, where $\xi \equiv 3 m-1$, and $j u n k(x) \in\{0,1\}^{n+\xi-1}$ is a certain bit string whose detail is irrelevant here. Note that $U$ consists of Clifford and $7(3 m-1)$ number of $T$ gates. (The 3 -CNF $g$ contains $2 m$ OR gates and $m-1$ AND gates. Each AND and OR gate can be simulated with a single Toffoli gate by using a single ancilla qubit. A single Toffoli gate can be simulated with Clifford and $7 T$ gates.) Let us consider the following circuit.

1. The initial state is $\left|0^{n}\right\rangle \otimes\left|0^{\xi}\right\rangle$. 
2. Apply $H^{\otimes n} \otimes I^{\otimes \xi}$ to obtain

$$
\frac{1}{\sqrt{2^{n}}} \sum_{x \in\{0,1\}^{n}}|x\rangle \otimes\left|0^{\xi}\right\rangle
$$

3. Apply $U$ to obtain

$$
\frac{1}{\sqrt{2^{n}}} \sum_{x \in\{0,1\}^{n}}|g(x)\rangle \otimes|j u n k(x)\rangle .
$$

4. Apply $Z \otimes I^{\otimes n+\xi-1}$ to obtain

$$
\frac{1}{\sqrt{2^{n}}} \sum_{x \in\{0,1\}^{n}}(-1)^{g(x)}|g(x)\rangle \otimes|j u n k(x)\rangle .
$$

5. Apply $U^{\dagger}$ to obtain

$$
\frac{1}{\sqrt{2^{n}}} \sum_{x \in\{0,1\}^{n}}(-1)^{g(x)}|x\rangle \otimes\left|0^{\xi}\right\rangle
$$

6. Apply $Z$ and $C Z$ that correspond to $f$ to obtain

$$
\frac{1}{\sqrt{2^{n}}} \sum_{x \in\{0,1\}^{n}}(-1)^{g(x)+f(x)}|x\rangle \otimes\left|0^{\xi}\right\rangle
$$

7. Apply $H^{\otimes n} \otimes I^{\otimes \xi}$ and measure all qubits in the first register in the computational basis.

This quantum computing uses $t \equiv 14(3 m-1)$ number of $T$ gates. The probability of obtaining $z \in\{0,1\}^{n}$ is

$$
p_{z}(f+g)=\left|\frac{1}{2^{n}} \sum_{x \in\{0,1\}^{n}}(-1)^{f(x)+\sum_{j=1}^{n} x_{j} z_{j}+g(x)}\right|^{2} .
$$

Assume that there exists a $T$-time classical probabilistic algorithm that outputs $z \in\{0,1\}^{n}$ with probability $q_{z}(f+g)$ such that

$$
\sum_{z \in\{0,1\}^{n}}\left|p_{z}(f+g)-q_{z}(f+g)\right| \leq \epsilon .
$$

From Markov's inequality,

$$
\operatorname{Pr}_{z}\left[\left|p_{z}(f+g)-q_{z}(f+g)\right| \geq \frac{\epsilon}{2^{n} \delta}\right] \leq \delta
$$

for any $f, g$, and $\delta>0$. According to the fine-grained Stockmeyer's theorem, a classical $O^{*}(T)$-time probabilistic algorithm that makes queries of length $O(T)$ to the NTIME $\left[n^{2}\right]$ oracle can compute $\tilde{q}_{z}(f+g)$ such that

$$
\left|q_{z}(f+g)-\tilde{q}_{z}(f+g)\right| \leq \xi q_{z}(f+g)
$$

where

$$
\xi \equiv \frac{2^{\frac{1}{\alpha}}-2^{-\frac{1}{\alpha}}}{2},
$$

for any $f, g$, integer $\alpha \geq 1$, and $z \in\{0,1\}^{n}$, with a success probability at least $w$. Due to the anti-concentration lemma [4]

$$
\operatorname{Pr}_{z, f}\left[p_{z}(f+g) \geq \frac{\tau}{2^{n}}\right] \geq \frac{(1-\tau)^{2}}{3}
$$


for any $0<\tau<1$.

Then we have

$$
\begin{aligned}
\left|p_{z}(f+g)-\tilde{q}_{z}(f+g)\right| & \leq\left|p_{z}(f+g)-q_{z}(f+g)\right|+\left|q_{z}(f+g)-\tilde{q}_{z}(f+g)\right| \\
& \leq\left|p_{z}(f+g)-q_{z}(f+g)\right|+\xi q_{z}(f+g) \\
& \leq\left|p_{z}(f+g)-q_{z}(f+g)\right|+\xi\left(p_{z}(f+g)+\left|p_{z}(f+g)-q_{z}(f+g)\right|\right) \\
& =\xi p_{z}(f+g)+\left|p_{z}(f+g)-q_{z}(f+g)\right|(1+\xi) \\
& \leq \xi p_{z}(f+g)+\frac{\epsilon}{2^{n} \delta}(1+\xi) \quad(\text { for at least } 1-\delta \text { fraction of } z) \\
& \left.\leq \xi p_{z}(f+g)+\sigma p_{z}(f+g)(1+\xi) \quad \text { for at least } \frac{\left(1-\frac{\epsilon}{\sigma \delta}\right)^{2}}{3} \text { fraction of }(z, f)\right) \\
& =p_{z}(f+g)(\sigma+(1+\sigma) \xi) \\
& \left.=p_{z}(f+g) u \text { (We take } u \equiv \sigma+(1+\sigma) \xi\right) .
\end{aligned}
$$

If we take $\epsilon$ and $\delta$ such that $-\delta+\frac{1}{3}\left(1-\frac{\epsilon}{\sigma \delta}\right)^{2}=v$, the above inequality is correct for at least $v$ fraction of $(z, f)$, which contradict Conjecture 3 .

\section{Discussion}

\subsection{Conjectures}

In this paper, we have shown additive-error fine-grained quantum supremacy based on several conjectures. In this subsection, we provide some "evidence" that support these conjectures.

Our conjectures are related to the exponential-time hypothesis (ETH) and the strong exponentialtime hypothesis (SETH) that are standard conjectures in fine-grained complexity theory $[25,26]$. $\mathrm{ETH}$ and SETH are stronger (or more pessimistic) versions of the famous $\mathrm{NP} \neq \mathrm{P}$ conjecture that says that an NP-complete problem cannot be solved in polynomial-time. More precisely, ETH is stated as follows:

Conjecture 4 (ETH) Any (classical) deterministic algorithm that decides whether $\# f>0$ or $\# f=0$ given (a description of) a 3-CNF with $n$ variables, $f:\{0,1\}^{n} \rightarrow\{0,1\}$, needs $2^{\Omega(n)}$ time. Here, $\# f \equiv \sum_{x \in\{0,1\}^{n}} f(x)$.

SETH is the stronger version of ETH which says as follows:

Conjecture 5 (SETH) Let $A$ be any (classical) deterministic $T(n)$-time algorithm such that the following holds: given (a description of) a CNF, $f:\{0,1\}^{n} \rightarrow\{0,1\}$, with at most cn clauses, $A$ accepts if $\# f>0$ and rejects if $\# f=0$, where $\# f \equiv \sum_{x \in\{0,1\}^{n}} f(x)$. Then, for any constant $a>0$, there exists a constant $c>0$ such that $T(n)>2^{(1-a) n}$ holds for infinitely many $n$.

All conjectures used in this paper are the average-case hardness of computing GapP functions within a multiplicative error in classical probabilistic time with an NTIME oracle, and therefore different from ETH and SETH. There are, however, three reasons that support these conjectures.

First, our conjectures consider GapP functions, while ETH and SETH consider \#P functions. A GapP function is a difference of two \#P functions. Furthermore, to our knowledge, only known way of computing a GapP function is to compute the number of accepting and rejecting paths (i.e., \#P functions). Therefore, computing GapP functions should not be easier than computing \#P functions.

Second, our conjectures study average cases. One might think that solving an average case could be easier than the worst case, but, at least, SETH has not been refuted even in average cases. (The best upper-bound is Ref. [27].)

Third, our conjectures allow the algorithm to use an NTIME oracle. We point out that at least \#ETH, which is the counting version of ETH, has not been refuted for MA (which is in ZPP ${ }^{\mathrm{NP}}$ ) and $\mathrm{AM}$ (which is coNP ${ }^{\mathrm{NP}}$ ).

It is an important open problem for the research of (not only fine-grained but also non-finegrained) quantum supremacy to show additive-error quantum supremacy based on standard conjectures. 


\subsection{Other models}

For simplicity, we have considered the three models, but similar results should hold for other sub-universal models such as the one-clean qubit model and the random circuit model. For all sub-universal models, Markov's inequality and the anti-concentration lemma hold. (For the Boson sampling model, the anti-concentration is a conjecture.) Therefore if we assume similar averagecase hardness conjectures as we have introduced in this paper, we should be able to show additiveerror fine-grained quantum supremacy for other sub-universal models.

\section{Acknowledgements}

TM thanks Yoshifumi Nakata for discussion. We thank authors of Ref. [14] for comments on our manuscript, and sharing their draft. We also thank anonymous reviewers, especially one reviewer who told us some errors and an improvement of fine-grained Stockmeyer's theorem. TM is supported by MEXT Q-LEAP, JST PRESTO No.JPMJPR176A, and the Grant-in-Aid for Young Scientists (B) No.JP17K12637 of JSPS. ST is supported by JSPS KAKENHI Grant Numbers $16 \mathrm{H} 02782,18 \mathrm{H} 04090$, and 18K11164.

\section{A Appendix}

In this Appendix, we provide a proof of the fine-grained Stockmeyer's theorem. The proof is a straightforward generalization of the one given in Ref. [28].

\section{A.1 A pairwise independent hash family and the leftover hash lemma}

To show the fine-grained Stockmeyer's theorem, we need the following two lemmas. Their proofs can be found in standard text books of complexity theory, such as Ref. [29].

Lemma 1 (A pairwise independent hash family) Let $A$ be a random $m \times n$ binary Toeplitz matrix, and $b$ be a random $m$-dimensional binary vector. (Here, a Toeplitz matrix is a matrix whose matrix elements satisfy $a_{i, j}=a_{i+1, j+1}$.) Then, the family $H \equiv\left\{h_{A, b}\right\}_{A, b}$ of functions, $h_{A, b}:\{0,1\}^{n} \rightarrow\{0,1\}^{m}$, with $h_{A, b}(x) \equiv A x+b$ satisfies

$$
\operatorname{Pr}_{A, b}\left[h_{A, b}\left(x_{1}\right)=y_{1} \wedge h_{A, b}\left(x_{2}\right)=y_{2}\right]=\frac{1}{2^{2 m}}
$$

for any $x_{1} \neq x_{2} \in\{0,1\}^{n}$ and $y_{1}, y_{2} \in\{0,1\}^{m}$.

Lemma 2 (The leftover hash lemma) Let $S \subseteq\{0,1\}^{n}$ be a set of $n$-bit strings. Then

$$
\operatorname{Pr}_{A, b}\left[||\left\{x \in S: h_{A, b}(x)=0^{m}\right\} \mid-\frac{|S|}{2^{m} \mid} \geq \epsilon \frac{|S|}{2^{m}}\right] \leq \frac{2^{m}}{\epsilon^{2}|S|}
$$

\section{A.2 Algorithm $A_{k}$}

In this subsection, we construct the algorithm $A_{k}$, which is used for fine-grained Stockmeyer's theorem. Let $G$ be an $n$-time deterministic classical algorithm. Let $S \equiv\left\{x \in\{0,1\}^{n}: G(x)=1\right\}$. Let $k$ be an integer such that $1 \leq k \leq n$. We construct a classical probabilistic $O(r n)$-time algorithm $A_{k}$ that gets a description of $G$ as the input, and that makes queries of length $O(n)$ to the NTIME $\left[n^{2}\right]$ oracle such that

- If $|S| \geq 2^{k+1}$ then $\operatorname{Pr}\left[A_{k}\right.$ accepts $] \geq 1-e^{-r}$.

- If $|S|<2^{k}$ then $\operatorname{Pr}\left[A_{k}\right.$ accepts $] \leq e^{-r}$.

To construct $A_{k}$, let us consider the following algorithm.

1. If $k \leq 5$, query the NTIME[n] oracle whether $|S| \geq 2^{k+1}$ or not. (The query to the oracle is the description of $G$. Given the description of $G$, deciding $|S| \geq 2^{k+1}$ or not is in NTIME $[n]$.) Accept if the oracle answer is yes. If the oracle answer is no, reject. 
2. If $k \geq 6$, set $m \equiv k-5$. Randomly choose an $m \times n$ binary Toeplitz matrix $A$, and an $m$-dimensional binary vector $b$. It takes $n+2 m-1=O(n)$ time. Define the function $h_{A, b}:\{0,1\}^{n} \rightarrow\{0,1\}^{m}$ by $h_{A, b}(x) \equiv A x+b$. Query the NTIME $\left[n^{2}\right]$ oracle whether $\left|\left\{x \in S: h_{A, b}(x)=0^{m}\right\}\right| \geq 48$ or not. (The query to the oracle is the description of $G, A$, and $b$. Given the description of $G, A$, and $b$, deciding $\left|\left\{x \in S: h_{A, b}(x)=0^{m}\right\}\right| \geq 48$ or not is in NTIME $\left[n^{2}\right]$.) If the oracle answer is yes, accept. If the oracle answer is no, reject.

Assume that $k \leq 5$. Then, if $|S| \geq 2^{k+1}$, the probability that the algorithm accepts is 1 . If $|S|<2^{k}$, the probability that the algorithm accepts is 0 .

Assume that $k \geq 6$. If $|S| \geq 2^{k+1}$, then $|S| \geq 2^{m+6}$ and therefore

$$
\begin{aligned}
\operatorname{Pr}[\text { reject }] & =\operatorname{Pr}_{A, b}\left[\left|\left\{x \in S: h_{A, b}(x)=0^{m}\right\}\right|<48\right] \\
& \leq \operatorname{Pr}_{A, b}\left[\left|\left\{x \in S: h_{A, b}(x)=0^{m}\right\}\right| \leq \frac{3}{4} \frac{|S|}{2^{m}}\right] \\
& =\operatorname{Pr}_{A, b}\left[-\left|\left\{x \in S: h_{A, b}(x)=0^{m}\right\}\right|+\frac{|S|}{2^{m}} \geq \frac{1}{4} \frac{|S|}{2^{m}}\right] \\
& \leq \operatorname{Pr}_{A, b}\left[\left|\left\{x \in S: h_{A, b}(x)=0^{m}\right\}\right|-\frac{|S|}{2^{m} \mid} \geq \frac{1}{4} \frac{|S|}{2^{m}}\right] \\
& \leq \frac{2^{m+4}}{|S|} \leq \frac{1}{4} .
\end{aligned}
$$

If $|S|<2^{k}$, define a superset $S^{\prime} \supseteq S$ with $\left|S^{\prime}\right|=2^{k}$. Then,

$$
\begin{aligned}
\operatorname{Pr}[\text { accept }] & =\operatorname{Pr}_{A, b}\left[\left|\left\{x \in S: h_{A, b}(x)=0^{m}\right\}\right| \geq 48\right] \\
& \leq \operatorname{Pr}_{A, b}\left[\left|\left\{x \in S^{\prime}: h_{A, b}(x)=0^{m}\right\}\right| \geq 48\right] \\
& =\operatorname{Pr}_{A, b}\left[\left|\left\{x \in S^{\prime}: h_{A, b}(x)=0^{m}\right\}\right| \geq \frac{3}{2} \frac{\left|S^{\prime}\right|}{2^{m}}\right] \\
& =\operatorname{Pr}_{A, b}\left[\left|\left\{x \in S^{\prime}: h_{A, b}(x)=0^{m}\right\}\right| \geq \frac{\left|S^{\prime}\right|}{2^{m}}+\frac{1}{2} \frac{\left|S^{\prime}\right|}{2^{m}}\right] \\
& =\operatorname{Pr}_{A, b}\left[\left|\left\{x \in S^{\prime}: h_{A, b}(x)=0^{m}\right\}\right|-\frac{\left|S^{\prime}\right|}{2^{m}} \geq \frac{1}{2} \frac{\left|S^{\prime}\right|}{2^{m}}\right] \\
& \leq \operatorname{Pr}_{A, b}\left[||\left\{x \in S^{\prime}: h_{A, b}(x)=0^{m}\right\}\left|-\frac{\left|S^{\prime}\right|}{2^{m}}\right| \geq \frac{1}{2} \frac{S^{\prime} \mid}{2^{m}}\right] \\
& \leq \frac{2^{m+2}}{\left|S^{\prime}\right|}=\frac{1}{8} .
\end{aligned}
$$

Therefore we have constructed the algorithm such that

- If $|S| \geq 2^{k+1}$ then $\operatorname{Pr}[$ accept $] \geq \frac{3}{4}$.

- If $|S|<2^{k}$ then $\operatorname{Pr}[$ accept $] \leq \frac{1}{8}$.

By repeating this algorithm $O(r)$ times, we get the final result.

\section{A.3 Fine-grained Stockmeyer's theorem}

Theorem 4 For any classical probabilistic T-time algorithm that outputs $z \in\{0,1\}^{N}$ with probability $q_{z}$, and any constant integer $\alpha \geq 1$, there exists a $O(T \log (T) \log \log (T))$-time classical probabilistic algorithm that makes queries of length $O(T)$ to the NTIME $\left[n^{2}\right]$ oracle that outputs $\tilde{q}_{z}$ such that

$$
\left|q_{z}-\tilde{q}_{z}\right| \leq \frac{2^{\frac{1}{\alpha}}-2^{-\frac{1}{\alpha}}}{2} q_{z}
$$

with a success probability at least $w$ for any $z \in\{0,1\}^{N}$. Here, $w$ is a certain constant. 
Proof of Theorem 4. Let $C$ be a $T$-time deterministic classical algorithm such that

$$
\frac{\left|\left\{r \in\{0,1\}^{T}: C(r)=z\right\}\right|}{2^{T}}=q_{z}
$$

for all $z \in\{0,1\}^{N}$. For each $z \in\{0,1\}^{N}$, let us define the set $S_{z} \subseteq\{0,1\}^{T}$ by

$$
S_{z} \equiv\left\{r \in\{0,1\}^{T}: C(r)=z\right\}
$$

For any integer $\alpha \geq 1$, define

$$
S_{z}^{\times \alpha} \equiv\left\{\left(r_{1}, \ldots, r_{\alpha}\right) \in\left(\{0,1\}^{T}\right)^{\times \alpha}: C\left(r_{1}\right)=\ldots=C\left(r_{\alpha}\right)=z\right\} .
$$

For $S_{z}^{\times \alpha}$, find an integer $\eta \in\{1,2, \ldots, \alpha T\}$ such that $A_{\eta-1}$ accepts and $A_{\eta}$ rejects by the binary search. It takes classical probabilistic $O(T \log (T) \log \log (T)$ )-time that makes queries of length $O(T)$ to the NTIME $\left[n^{2}\right]$ oracle. (For the binary search, $O(\log T)$ steps are necessary, and each implementation of the algorithm $A$ takes $O(T \log \log (T))$ steps.) Then, $2^{\eta-1}<\left|S_{z}^{\times \alpha}\right|<2^{\eta+1}$ with a sufficiently small failure probability.

If we define $\sigma \equiv 2^{\frac{\eta}{\alpha}}, \frac{1}{2} \sigma^{\alpha}<\left|S_{z}\right|^{\alpha}<2 \sigma^{\alpha}$. Hence $\left(\frac{1}{2}\right)^{\frac{1}{\alpha}} \sigma<\left|S_{z}\right|<2^{\frac{1}{\alpha}} \sigma$. If we define $\tilde{q}_{z} \equiv \sigma / 2^{T}$, we obtain the result.

\section{References}

[1] B. M. Terhal and D. P. DiVincenzo, Adaptive quantum computation, constant depth quantum circuits and Arthur-Merlin games. Quant. Inf. Comput. 4, 134 (2004). DOI:10.26421/QIC4.2

[2] S. Aaronson and A. Arkhipov, The computational complexity of linear optics. Theory of Computing 9, 143 (2013). DOI:10.1145/1993636.1993682

[3] M. J. Bremner, R. Jozsa, and D. J. Shepherd, Classical simulation of commuting quantum computations implies collapse of the polynomial hierarchy. Proc. R. Soc. A 467, 459 (2011). DOI:10.1098/rspa.2010.0301

[4] M. J. Bremner, A. Montanaro, and D. J. Shepherd, Average-case complexity versus approximate simulation of commuting quantum computations. Phys. Rev. Lett. 117, 080501 (2016). DOI:10.1103/PhysRevLett.117.080501

[5] E. Knill and R. Laflamme, Power of one bit of quantum information. Phys. Rev. Lett. 81, 5672 (1998). DOI:10.1103/PhysRevLett.81.5672

[6] T. Morimae, K. Fujii, and J. F. Fitzsimons, Hardness of classically simulating the one clean qubit model. Phys. Rev. Lett. 112, 130502 (2014). DOI:10.1103/PhysRevLett.112.130502

[7] T. Morimae, Hardness of classically sampling one clean qubit model with constant total variation distance error. Phys. Rev. A 96, 040302(R) (2017). DOI:10.1103/PhysRevA.96.040302

[8] K. Fujii, H. Kobayashi, T. Morimae, H. Nishimura, S. Tamate, and S. Tani, Impossibility of classically simulating one-clean-qubit model with multiplicative error. Phys. Rev. Lett. 120, 200502 (2018). DOI:10.1103/PhysRevLett.120.200502

[9] K. Fujii, H. Kobayashi, T. Morimae, H. Nishimura, S. Tamate, and S. Tani, Power of quantum computation with few clean qubits. Proceedings of 43rd International Colloquium on Automata, Languages, and Programming (ICALP 2016), pp.13:1-13:14 (2016). DOI:10.4230/LIPIcs.ICALP.2016.13

[10] T. Morimae, Y. Takeuchi, and H. Nishimura, Merlin-Arthur with efficient quantum Merlin and quantum supremacy for the second level of the Fourier hierarchy. Quantum 2, 106 (2018). 10.22331/q-2018-11-15-106

[11] A. Bouland, B. Fefferman, C. Nirkhe, and U. Vazirani, On the complexity and verification of quantum random circuit sampling. Nature Phys. 15, 159 (2019). DOI:10.1038/s41567-0180318-2 
[12] R. Movassagh, Efficient unitary paths and quantum computational supremacy: A proof of average-case hardness of Random Circuit Sampling. arXiv:1810.04681

[13] R. Movassagh, Cayley path and quantum computational supremacy: A proof of average-case \#P-hardness of Random Circuit Sampling with quantified robustness. arXiv:1909.06210

[14] A. M. Dalzell, A. W. Harrow, D. E. Koh, and R. L. La Placa, How many qubits are needed for quantum computational supremacy? Quantum 4, 264 (2020). DOI:10.22331/q-2020-05-11-264

[15] A. M. Dalzell, Bachelor thesis, MIT (2017). https://dspace.mit.edu/handle/1721.1/111859

[16] C. Huang, M. Newman, and M. Szegedy, Explicit lower bounds on strong quantum simulation. arXiv: 1804.10368

[17] C. Huang, M. Newman, and M. Szegedy, Explicit lower bounds on strong simulation of quantum circuits in terms of $T$-gate count. arXiv:1902.04764

[18] T. Morimae and S. Tamaki, Fine-grained quantum computational supremacy. Quant. Inf. Comput. 19, 1089 (2019). DOI:10.26421/QIC19.13-14

[19] R. Hayakawa, T. Morimae, and S. Tamaki, Fine-grained quantum supremacy based on Orthogonal Vectors, 3-SUM and All-Pairs Shortest Paths. arXiv:1902.08382

[20] R. R. Williams, Strong ETH breaks with Merlin and Arthur: short non-interactive proofs of batch evaluation. Proceedings of the 31st Conference on Computational Complexity (CCC'16), pages 1-17 (2016). DOI:10.4230/LIPIcs.CCC.2016.2

[21] Dell and Lapinskas, Fine-grained reductions from approximate counting to decision. Proceedings of the 50th Annual ACM SIGACT Symposium on Theory of Computing (STOC 2018), pages 281-288 (2018). 10.1145/3188745.3188920

[22] R. Williams, Counting solutions to polynomial systems via reductions. Proceedings of the 1st Symposium on Simplicity in Algorithms (SOSA 2018). DOI:10.4230/OASIcs.SOSA.2018.6

[23] D. Lokshtanov, R. Paturi, S. Tamaki, R. Williams, and H. Yu, Beating brute force for systems of polynomial equations over finite fields. Proceedings of the 28th Annual ACM-SIAM Symposium on Discrete Algorithms, pp.2190-2202 (2017). DOI:10.1137/1.9781611974782.143

[24] A. Cosentino, R. Kothari, and A. Paetznick, Dequantizing read-once quantum formulas. arXiv:1304.5164; 8th Conference on the Theory of Quantum Computation, Communication and Cryptography (TQC 2013), Leibniz International Proceedings in Informatics (LIPIcs) 22, pp. 80-92 (2013). 10.4230/LIPIcs.TQC.2013.80

[25] R. Impagliazzo, R. Paturi, and F. Zane, Which problems have stronly exponential complexity? J. Comput. Syst. Sci. 63(4), 512-530 (2001). DOI:10.1006/jcss.2001.1774

[26] R. Impagliazzo and R. Paturi, On the complexity of k-SAT. J. Comput. Syst. Sci. 62(2), 367-375 (2001). DOI:10.1006/jcss.2000.1727

[27] A. Lincoln and A. Yedidia, Faster random $k$-CNF satisfiability. 47th International Colloquium on Automata, Languages, and Programming (ICALP 2020). DOI:10.4230/LIPIcs.ICALP.2020.78

[28] L. Trevisan, Lecture notes on computational complexity.

[29] O. Goldreich, Computational Complexity: a conceptual perspective. Cambridge University Press (2008). 